Décadrages Décadrages

cinéma, à travers champs Cinéma, à travers champs

15 | 2009

Raoul Ruiz

\title{
Tres Tristes Tigres, fourchelangues et contes à rebours
}

\section{François Bovier}

\section{(2) OpenEdition}

1 Journals

Édition électronique

URL : https://journals.openedition.org/decadrages/133

DOI : $10.4000 /$ decadrages. 133

ISSN : 2297-5977

Éditeur

Association Décadrages

Édition imprimée

Date de publication : 1 octobre 2009

Pagination : $57-68$

ISBN : 978-2-9700668-0-4

ISSN : 2235-7823

Référence électronique

François Bovier, «Tres Tristes Tigres, fourchelangues et contes à rebours », Décadrages [En ligne], 15। 2009, mis en ligne le 26 novembre 2012, consulté le 03 avril 2022. URL : http://

journals.openedition.org/decadrages/133; DOI : https://doi.org/10.4000/decadrages.133

(B) Décadrages 


\section{Tres Tristes Tigres, fourchelangues et contes}

\section{à rebours}

\section{par François Bovier}

Le premier long métrage de Raùl Ruiz (le cinéaste n’a pas encore françisé son prénom): Tres Tristes Tigres (Trois tristes tigres, Chili, 1968, 105 min.), qui a remporté en 1969 le Léopard d'or à Locarno, est étroitement associé au renouvellement du cinéma chilien $\mathbf{1}$. Parallèlement, il est tentant de soutenir que Tres Tristes Tigres participe à l'élaboration d'un univers «baroque $\mathbf{2}$ qui caractériserait les films de la maturité du cinéaste - tout au moins, si l'on s'en tient à la réception critique de Ruiz en France, salué dans son pays d'adoption, au début des années 1980, comme un «auteur» incontournable qui institue au cinéma une «esthétique du simulacre»3.

D’emblée, pour éviter l'équivoque, il convient de préciser que le terme «baroque» désigne en ce cas un récit qui présente simultanément une multiplicité de sens incompossibles, plutôt qu'un mode de représentation affirmant son artificialité, procédant par surcharge d'ornements ou de formes finement ciselées. Christine Buci-Glucksmann, en faisant

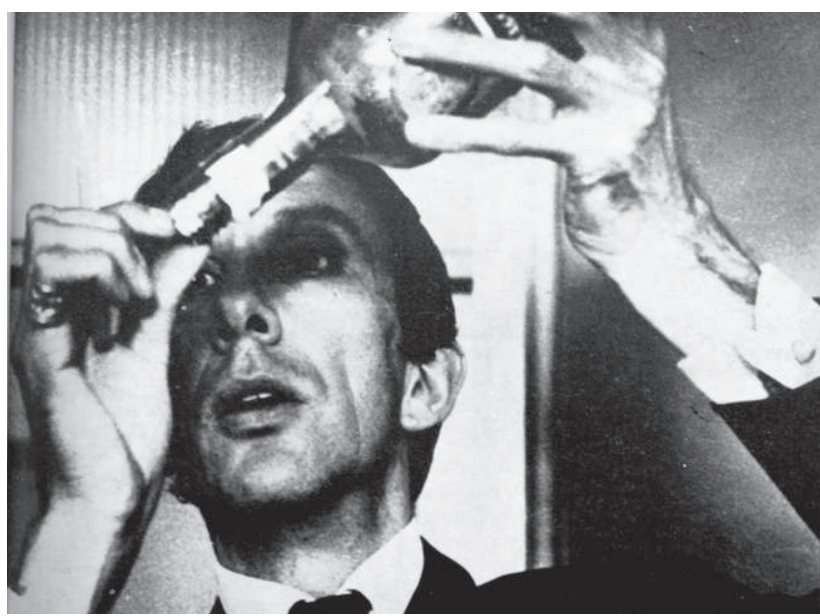

1 Voir, par exemple, Zuzana M. Pick, "Chilean Cinema: Ten Years of Exile (1973-1983) " in Jump Cut, n 32 , avril 1987, pp. 66-70: "La présentation de quatre longs-métrages au deuxième Festival de Vina del Mar en 1969 a marqué les débuts du (nouveau cinéma) au Chili. Valparaiso mi amor (Valparaiso mon amour, 1969) de Aldo Francia, El Chacal de Nahueltoro (Le Chacal de Nahueltoro, 1969) de Miguel Littin, Caliche Sangriento (Salpêtre sanglant, 1968) de Helvio Soto et Tres Tristes Tigres (Trois Tristes Tigres, 1968) de Raùl Ruiz sont apparus comme le paradigme d'un nouveau mode de production de longs métrages dans un pays sur le point d'accomplir une révolution politique unique. [...] Raùl Ruiz est le cinéaste chilien le plus prolifique. Prodigieux narrateur et créateur d'images, son travail se distingue par une expérimentation originale et personnelle sur les modalités de la représentation et les formes du discours. Depuis Tres Tristes Tigres, son approche novatrice du cinéma ainsi que son indépendance et sa position critique à l'égard du réductionnisme politique l'ont distingué de la plupart des autres cinéastes latino-américains. " (Nous traduisons).

2 Sur l'esthétique baroque de Ruiz, voir Christine Buci-Glucksmann, Fabrice Revault d'Allonnes, Raoul Ruiz, Editions Dis Voir (Série Entrevues), Paris, 1987.

3 Pascal Bonitzer ("R.R. ou l'art du faux: Métamorphoses", in Cahiers du cinéma, spécial Raoul Ruiz, $n^{\circ} 345$, mars 1983, p. 21) inscrit l'univers filmique de Ruiz dans une "esthétique du simulacre": "Les trucages, multiples [...], ne se dissimulent pas, l'image est donnée pour ce qu'elle est: une image, c'est-à-dire une illusion, un trompe-l'œil, mais aussi une sorte de poison, qui contamine le corps qu'elle reflète, à moins qu'elle ne soit justement, de ce corps opaque, inconnaissable, de cette chose-ensoi, l'émanation vénéneuse, le vêtement mortel. " Dans ce numéro spécial des Cahiers du cinéma, les critiques de Pascal Bonitzer, Serge Daney, Yann Lardeau, Danièle Dubroux, Louis Skorecki, Michel Chion et, dans une moindre mesure, les témoignages des opérateurs Henri Alekan et Sacha Vierny ou encore l'entretien accordé par Ruiz, se focalisent sur les films français suivants: L'Hypothèse du tableau volé (France, 1978), Le Borgne (France, 1980), Le Toit de la baleine (Hollande/France, 1981), Les Trois Couronnes du matelot (France/Chili, 1982). 
4 Voir Walter Benjamin, Origine du drame baroque allemand, Flammarion, Paris, 1985 [première édition: Ursprung des deutschen Trauerspiels, Ernst Rowohlt, Berlin, 1928].

5 Christine Buci-Glucksmann, "L'œil baroque de la caméra", dans Christine Buci-Glucksmann, Fabrice Revault d'Allonnes, Raoul Ruiz, op. cit., pp. 16-17.

6 Raoul Ruiz, Poétique du cinéma 1 (Miscellanées), Editions Dis Voir, Paris, 1995, p. 80.

7 Adrian Martin, "Never One Space: an Interview with Raùl Ruiz", in Cinema Papers, n 91, janvier 1993, cité dans Adrian Martin, "Raùl Ruiz", $11^{e}$ Biennale de l'Image en Mouvement, Centre pour I'Image Contemporaine, Genève, 2005, p. 45.

8 Alejo Carpentier, "Le réel merveilleux américain ", Chroniques, Gallimard, Paris, 1983, p. 344 [première édition: "Lo real Maravilloso in America", El Nacianal, 8 avril 1948, republié dans la préface de El Reino de este mundo, Edicion y Distribucion Iberoamericana, Mexico, 1949]. référence à l'«élan allégorique» $\mathbf{4}$ qui impulse la logique narrative du drame baroque allemand, peut ainsi écrire:

"Tel serait donc ce modèle enfin trouvé du film ruizien: non pas l'enquête policière traditionnelle avec sa vérité dramatique, sa progression, ses personnages, mais l'enquête baroque, celle dans laquelle la modernité s'est créée en instituant l'ambiguïté, la polysémie du réel et du récit: Don Quichotte. De la folie, des visions et < fantastiqueries ' de ce chevalier errant ne naît jamais une vérité ultime, mais une multiplicité interprétative répondant aux niveaux de récit, aux points de vue adoptés, à l'angle de vue sur le monde.»5

Et de fait, comme Ruiz l'explicite par la suite, ses films alternent entre différents niveaux de signification, sans accorder de primauté à aucun d'entre eux :

«Il arrive que dans mes projets je cherche à passer d'un monde à un autre, utilisant une technique décrite dans la Venise baroque comme ‘Il Ponte`, une manière de produire des agents anamorphiques qui jouent avec les quatre niveaux de la rhétorique médiévale : littérale, allégorique, éthique et anagogique. [...] A ceci près que, plutôt que de chercher à lire les quatre niveaux à la fois, le but est de passer constamment d'un niveau à un autre.» 6

Ce modèle est encore réfracté à travers la culture chilienne, dont on ne peut dissocier les premiers films de Ruiz:

"J'étais simplement fasciné par ma propre culture, la culture latinoaméricaine, qui a la particularité d'avoir commencé à l'époque du baroque en Espagne. Et c'est vrai qu'il me semble qu'aujourd'hui la plupart des arts ont rejeté cette forme d'allégorie qui a joué un rôle si important dans l'histoire de la culture. » $\mathbf{7}$

Plus précisément, on peut affirmer que le cinéma de Ruiz s'inscrit dans le registre du "réalisme merveilleux", tel que l'a défini Alejo Carpentier : "[...] le merveilleux commence à l'être de façon évidente lorsqu'il surgit d'une altération inattendue de la réalité (le miracle), d'une révélation privilégiée de la réalité, d'une illumination inhabituelle ou qui favorise singulièrement les richesses inaperçues de la réalité, d'un élargissement des échelles et des catégories de la réalité, perçues avec une particulière intensité en vertu d'une exaltation de l'esprit qui le conduit à une sorte d'état limite`. Pour commencer, la sensation du merveilleux présuppose une foi. » $\mathbf{8}$

Selon cette perspective, Tres Tristes Tigres, long métrage dont la structure narrative est pour le moins oblique, acquiert un statut de geste inaugural dans une stratégie de leurres et de masques qui définit le style de Ruiz. Ce style, soulignons-le, rompt avec la reconduction des conventions de 
la peinture baroque et plus radicalement encore avec la belle image. Ruiz peut ainsi envisager son premier long-métrage comme une œuvre anti-

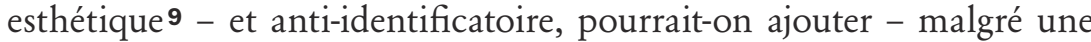
attention indéniable consacrée à la composition du cadre et à la découpe de l'espace (suscitant une tension entre l'inscription des personnages ou des objets à l'écran et leur basculement hors-champ, notamment à travers les mouvements de recadrage de la caméra).

Mais l'on peut tout aussi bien soutenir que Tres Tristes Tigres - film peu commenté $\mathbf{1 0}$, qui résiste singulièrement à l'analyse - n'a, somme toute, pas grand-chose à voir avec l'«élan allégorique» décrit par Benjamin. La relation à un mode d'écriture par rébus et constante bifurcation du sens est encore problématisée par la portée géopolitique du film. Et sur ce point encore, il est loin d'être certain que les fictions chiliennes de Ruiz contournent productivement la censure d'Etat en recourant à l'expression par la parabole. Rappelons, en premier lieu, que Tres Tristes Tigres n'est pas réalisé sous la dictature. Le titre du film, qui renvoie explicitement à un fourchelangue, une formule enfantine enchaînant les assonances et les allitérations, au point de faire fourcher la langue des locuteurs (jeu sur la dimension sonore des mots inscrit dans la première scène tournée du film - nous y reviendrons), peut tout au plus s'entendre comme une référence à la situation du Chili, à la fin des années 1960 : c'est-à-dire au système dit des «trois tiers» (los tres tercios), une alliance plutôt bancale entre la droite, le Parti démocrate-chrétien et le front de l'Unité populaire11, sous la présidence d'Eduardo Frei. Les dialogues du film évoquent par ailleurs le gouvernement du président chilien :

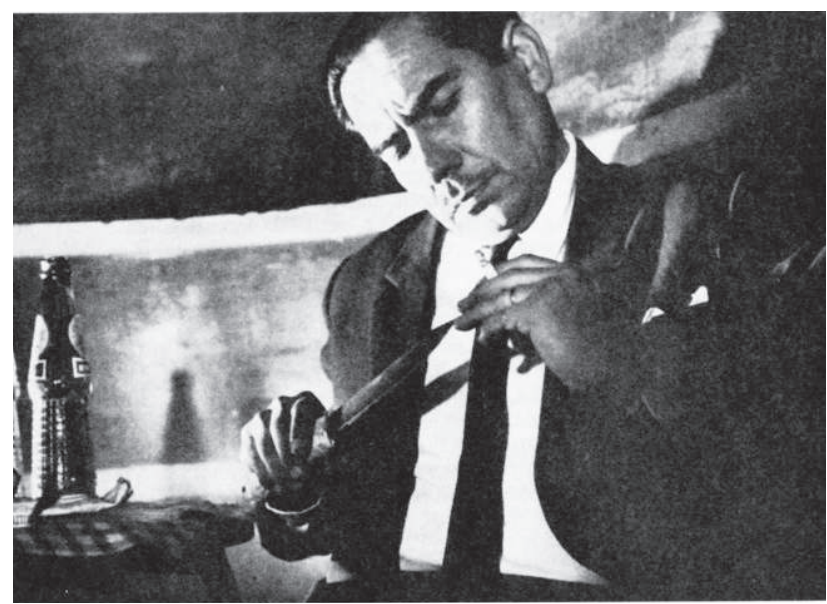

9 "Cette obsession de ne pas faire d'esthétisme vient aussi de discussions sur le baroque. [...] Comme tout peut devenir baroque, il faut trouver l'axe à partir duquel ce qui est beau va cesser d'être beau pour devenir excessif." Juste auparavant, Ruiz prévient: "De là, cette volonté de jouer avec ce qui est moche, et plus encore ce qui est plat, puisque que c'est la seule chose qui soit neutre." (Benoît Peeters, "Les années chiliennes. Entretien avec Raoul Ruiz", in Théâtres au cinéma, n 14, 2003, p. 21).

10 Relevons que parmi les rares critiques français à s'être exprimés sur Tres Tristes Tigres au moment de sa sortie, Louis Seguin souligne le classicisme et l'"humour fou " du film: "Le sujet, aussi bien que le langage, de Tres Tristes Tigres sont des plus classiques. Ils renvoient à ce réalisme, mélange d'attirance maniaque pour la platitude, voire le sordide [...]. " (in Positif, $n^{\circ} 112$, janvier 1970).

11 Ruiz milite au sein du front de I'Unité populaire; sous le gouvernement socialiste d'Allende, il sera responsable du département cinéma; suite au coup d'Etat de Pinochet, le 11 septembre 1973, il est contraint à l'exil avec sa femme Valeria Sarmiento, comme nombre d'artistes et intellectuels chiliens. Les films chiliens de Ruiz évoquent régulièrement la situation politique du pays, comme le réalisateur le souligne: "Au Chili il y avait de tout. Des maoïstes, des trotskystes ou même des sociaux-démocrates, partisans de la lutte armée. On pouvait voir des sociaux-démocrates lancer des propos maoïstes, à côté de maos tout à fait raisonnables et frileux. Toute une ébullition politique très proche de l'ébullition artistique. Evidemment, on retrouve de cela dans mes films chiliens." ("Entretien avec Raoul Ruiz", dans Christine Buci-Glucksmann, Farbrice Revault d'Allonnes, Raoul Ruiz, op. cit., p. 93). 
12 Voir Alejandro Sieveking, Tres obras de teatro: La remolienda, Tres tristes tigres, La mantis religiosa, Editorial Universitaria, Santiago, 1974 [première édition de Tres Tristes Tigres: 1967]. Ruiz s'éloigne considérablement de la situation mise en scène dans la pièce de Sieveking: "J'avais bien aimé le rapport aux comédiens que la pièce induisait, la liberté qu'elle pouvait laisser, mais j'avais moins aimé certains aspects un peu sentimentaux que j'ai essayé de gommer" (Benoît Peeters, "Les années chiliennes. Entretien avec Raoul Ruiz", op. cit., p. 18). II peut ainsi introduire un nouveau personnage central, Don Luis Obeda. Un certain nombre de dialogues du film respecte néanmoins les échanges de paroles dans la pièce. Le titre du film peut encore évoquer l'ouvrage homonyme de Guillermo Cabrera Infante (Tres Tristes Tigres, Seix Barral, Barcelone, 1967 [Trois Tristes Tigres, Gallimard, Paris, 1989]), qui multiple les jeux de mots, les calembours, les assonances et allitérations.

13 Benoît Peeters, "Les années chiliennes. Entretien avec Raoul Ruiz", op. cit., p. 18.

\section{4 lbid.}

15 Waldo Rojas présente Le Tango du veuf, œuvre inachevée de Ruiz, comme un film "suffisamment important pour régler certains comptes à ce penchant mal avoué des Chiliens pour le surréalisme ou plutôt pour le 'surréachilisme" "Waldo Rojas, "Images de passage ", in Théâtres au cinéma, op. cit., p. 26).

16 "Le principe actif du réaliste pudique consistait à considérer la notion de réalité, non plus comme une donnée toute faite, ni comme la découverte suprême, sublunaire et imperturbable, mais comme un système de dissimulations: la nature aime se cacher. [...] L'étape suivante fut l'abandon définitif du titre d'artistes et d'intellectuels pour ne devenir que de simples cclients de bar. Plus tard, aux alentours de 1969, et conséquence de tout cela, naquit ce film 'surprenant et polémique): Trois Tristes Tigres, annoncé comme l'œuvre mettant fin à la préhistoire cinématographique au Chili." (Waldo Rojas, op. cit., p. 27).

$\mathbf{1 7}$ "Ce qui m'intéresse dans le surréalisme c'est le côté tarte à la crème, décoratif, le stéréotype: tout ce qui a été récupéré par Dali, par la publicité. Je ne suis pas du tout convaincu par la métaphysique surréaliste." ("Entretien avec Raoul Ruiz", dans Christine Buci-Glucksmann, Fabrice Revault d'Allonnes, Raoul Ruiz, op. cit., p. 103).

18 /d., p. 87. dans un café, des hommes qui commencent par critiquer Eduardo Frei, se présentent ensuite comme faisant partie du gouvernement; l'un d'entre eux frappe au visage le protagoniste du film qui refusait de participer à la discussion; dans les toilettes, ils font mine de le passer à tabac. Mais il est fort malaisé de désigner, dans l'univers filmique de Tres Tristes Tigres, un représentant de ces différentes orientations politiques, tant la fonction et le rôle des personnages sont instables. En somme, Tres Tristes Tigres ne participe que lointainement au régime de l'«enquête baroque»: dans ce film, il n'y a nulle énigme à résoudre, nulle allégorie à reconstituer. Le mode "baroque", si tant est que l'on puisse déjà se référer à ce modèle, est pour le moins évidé, désublimé, désincarné.

Le film n'en répond pas moins à une méthode: adaptation libre d'une pièce de théâtre d'Alejandro Sieveking ${ }^{12}$, Tres Tristes Tigres repose sur des dialogues écrits et sur un dispositif de mise en situation d'acteurs, qui sont pour la plupart amateurs. Ruiz le soulignera lui-même: «Tout était écrit. Tout était prémédité. »13 Et le réalisateur d'enchaîner sur une remarque qui révèle bien le paradoxe de son écriture filmique, qui se désagrège à force de maîtrise et de surdétermination: "[...] on me demande régulièrement de faire des films écrits, sans se rendre compte que ce sont ceux-là, chez moi au moins, qui ont la structure la plus éclatée »14.

En ce sens, l'univers fictionnel de Tres Tristes Tigres se situe aux antipodes de l'écriture automatique. Comme le poète Waldo Rojas le fait remarquer, Ruiz et la génération d'artistes à laquelle il appartient s'inscrivent en porte-à-faux vis-à-vis du surréalisme ${ }^{\mathbf{1 5}}$. Plus adéquatement, Rojas réinscrit Tres Tristes Tigres dans le mode d'un singulier «réalisme pudique", qui dénaturalise les aspects les plus insignifiants de l'univers quotidien 16. Par la suite, Ruiz conforte cette position en opposant à la poétique des "signes ascendants» (au sens d'André Breton) et au mysticisme fin-de-siècle du surréalisme $\mathbf{1 7}$ un "réalisme fantastique», qui peut être spécifié comme un phénomène de suspension et de défamiliarisation de la perception:

«La quotidienneté comme source d'étrangeté, c'est le propre de l'Amérique latine. On pourrait parler de 〈réalisme fantastique〉 [lo real maravilloso], selon le titre du manifeste d'Alejo Carpentier. Pour lui, le fantastique est dans le paysage; mais au-delà, il est dans la réalité discontinue.»18

\section{L'éclatement du texte filmique}

Le point de départ de Tres Tristes Tigres est comparable, à plus d'un égard, à la séquence inaugurale de Shadows (Etats-Unis, 1957/1959), du moins dans la première version de Cassavetes (par la suite, Cassavetes linéa- 
rise son film, notamment en retournant certains plans $\left.{ }^{19}\right)$ : une scène prétexte, largement improvisée, détermine les relations entre les personnages et la tournure des évènements $\mathbf{2 0}$. En effet, dans un entretien contemporain à la sortie du film en France, Ruiz indique que le point de départ de Tres Tristes Tigres repose sur la séquence où les trois personnages principaux, à savoir Amanda, son frère Tito et son patron Rudi, sont dans une chambre:

"L'œuvre originale était un mélodrame d'un intérêt relatif dans le théâtre chilien et contenait une grande richesse de nuances, de situations et d'attitudes qui étaient l'apport direct des acteurs. Ceci était particulièrement évident dans une scène, celle que je pris pour la développer. Le reste fut un peu la contrepartie de cette scène qui était le nœud mélodramatique de la pièce de théâtre: un frère par ambition pratiquement prostitue et vend sa sœur. Mon travail consista précisément à faire la contrepartie de ceci, à placer le mélodrame au troisième plan et à donner une échelle possible de valeurs à cet acte, le convertissant en une chaîne d'événements, liés les uns aux autres (bien que cela ne soit pas toujours fait d'une façon aussi directe), de sorte que cet acte apparaisse comme le résultat d'un univers remis continuellement en jeu.» $\mathbf{2 1}$

Premier écart par rapport à la méthode d'improvisation élaborée par Cassavetes: Ruiz rejette la rencontre entre les protagonistes dans la seconde partie du film, rendant les interactions entre les personnages difficilement intelligibles. Second écart, qui achève de perdre le spectateur dans les méandres d'un texte filmique qui emprunte avec désinvolture

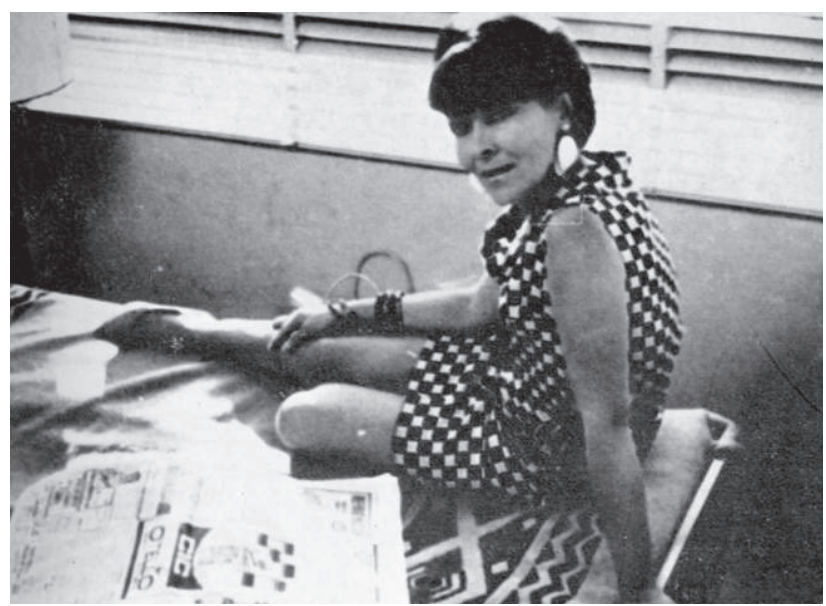

19 La première version de Shadows est vraisemblablement perdue. Jonas Mekas, ardent défenseur de la méthode d'improvisation de Cassavetes, se retournera contre celui-ci, dénonçant l'affadissement du propos dans la copie du film distribuée commercialement: "Je n'ai plus de doute sur le fait que, tandis que la seconde version de Shadows n'est qu'un film hollywoodien de plus - inspiré, cependant, par moments -, la première version est le film américain qui a le plus fait bouger les choses depuis dix ans. [...] Shadows rompt avec le cinéma officiel joué, avec les visages maquillés, avec les dialogues écrits, avec les scénarios à intrigue. [...] En attendant, la version abâtardie va être envoyée dans les festivals et promue officiellement, tandis que le vrai film, le premier Shadows, va être traité en enfant bâtard." (Jonas Mekas, "Deux versions de Shadows", Ciné-Journal. Un nouveau cinéma américain, 1959-1971, Paris Expérimental, Paris, pp. 28-29 [Village Voice, 27 janvier 1960]).

20 Cassavetes explicite en ces termes la genèse de son film: "Lors d'un cours, je fus tellement impressionné par une improvisation que je dis: 'Voilà qui ferait un film fantastique.' II s'agissait d'une fille noire qui passe pour une Blanche et perd son amant blanc lorsque celuici rencontre son frère noir." "Playboy Interview: John Cassavetes", in Playboy, octobre 1971]. Comme le note Nicole Brenez, qui cite l'entretien de Cassavetes, la scène à l'origine du film est aussi la première tournée: "il s'agit de la rencontre entre Hugh et Tony, c'est-àdire un Noir et un sectaire [bigot] [...]. En huit semaines, avec une caméra Arriflex 16mm, une pellicule ultra-rapide, quelques projecteurs, un système sonore Pedectone, ils tournent trente heures de rushes [...]." (Nicole Brenez, Shadows. John Cassavetes, Nathan, coll. Synopis, Paris, 1996, p. 33).

21 Federico de Cardenas, "Entretien avec Raùl Ruiz (Trois tristes tigres) ", in Positif, $n^{\circ} 123$, janvier 1971, p. 17. 
22 Amanda se présente à Rudi comme une actrice qui cherche du travail, fonction associée dans le film au strip-tease. Rudi relate ses amours d'antan (il a rompu avec une blonde intellectuelle, car elle palabrait sur la guerre du Vietnam) ou encore rapporte une histoire salace (une jeune fille prétendument vierge se présente aux portes du paradis, un ange médecin distingue sept égragnitures, et celle-ci répond à Saint-Pierre qui lui demande de décliner son identité: Blanche-Neige), avant de vouloir prouver à Amanda qu'il est un bon parti au lit. Le tout arrosé de force libations.

23 Federico de Cardenas, "Entretien avec Raùl Ruiz (Trois tristes tigres)", op. cit., p. 17. les conventions du film policier: les personnages sont absents à la scène ou, plus justement, l'interprètent sur un mode dédramatisé, en multipliant les sous-entendus et les jeux de mots. Le point de départ de l'intrigue, qui se joue dans une séquence d'environ dix minutes - rejetée, on l'a dit, au milieu du film -, d'abord découpée en champs-contrechamps (sur Rudi et Amanda), puis déstabilisée par les mouvements incessants d'une caméra qui navigue à vue entre les personnages, est défamiliarisé : les quiproquos entretenus par Amanda, les digressions, les anecdotes et les allusions grivoises de Rudi, tandis que Tito s'enivre ${ }^{22}$, brouillent la dramaturgie bien ordonnée de la pièce de Sieveking. Lorsque la formule «attrape-mots» à laquelle fait allusion le titre du film (et que l'on chercherait en vain dans la scène que Ruiz ici adapte) est déclinée avec peine par les protagonistes, la caméra réinscrit dans le cadre Tito, exclu des plans qui précédent, à travers un balaiement triangulaire de l'espace qui finit par se fixer sur le «couple» nouvellement formé par Rudi/ Amanda. A la différence du film de Cassavetes, l'enjeu de Tres Tristes Tigres se noue donc autour de rapports de classes (patron/employé, richesse/prostitution), et non de races. Mais l'improvisation des acteurs, à partir d'un texte écrit, règne : les gestes, les attitudes et les postures corporelles, tout comme les échanges de paroles, sont comme impulsés par la consommation d'alcool. La citation incomplète d'un fourchelangue («Tres tristes tigres tragan trigo en un trigal»), signalant au spectateur la centralité de la séquence, thématise ainsi l'incohérence et la gratuité du discours, dicté par les lapsus, les witz et les bons mots.

Le genre du film noir, auquel Tres Tristes Tigres fait lointainement écho, est ainsi prétexte à l'improvisation et à la dérive, contrecarrant la logique de la causalité, relâchant les relations de cause à effet et le bon enchaînement entre la situation et les actions. Il s'agit bien d'une méthode de jeu, comme le laisse entendre Ruiz:

«On renversait d'une certaine façon ce que nous voyions à travers le comportement des personnages; pour donner un exemple, un personnage va en poignarder un autre sans le regarder en face, contrairement à ce que ferait un 〈dur〉 normal.» $\mathbf{2 3}$

La perte de repères du spectateur gagne déjà les personnages : leur identité est singulièrement instable; les liens sociaux, les relations de travail ou d'amitié qu'ils entretiennent les uns à l'égard des autres font l'objet d'une constante et obscure négociation; la motivation de leurs actes leur demeure étrangère, comme s'ils subissaient un jeu dont ils ne connaissaient pas les règles.

Le travail sur la caméra est déterminant dans cette irrésolution de la fonction des personnages, la victime pouvant soudainement se transfor- 
mer en bourreau, l'employé se rebeller contre son patron et l'humilier à son tour. Les brusques mouvements de la caméra portée à l'épaule déstabilisent la construction du cadre, niant tout point d'arrimage et de de fixation du regard. Le film, qui adopte comme largeur standard le plan moyen, en ayant exclusivement recours à un objectif de $28 \mathrm{~mm}^{\mathbf{2 4}}$, ne cesse de décentrer et décadrer l'image, exploitant productivement le hors-champ 25. Ruiz a délibérément opté pour ce dispositif, le cadre apparaissant comme un espace contraignant qui menace cependant à tout instant de se dérober; cette dynamique de présence et d'absentement de la caméra surdétermine le jeu des acteurs, ceux-ci faisant l'expérience d'une confrontation concrète avec l'objectif de la caméra. La relation des acteurs à la caméra constitue l'une des contraintes que Ruiz s'est donné dans ce film, en étroite concertation avec l'opérateur Diego Bonacina:

"L'objectif se fixait sur l'acteur, provoquant une sensation physique gênante, pareille à celle que nous pouvons ressentir quand on nous passe les mains sur le visage. [...] L'acteur a alors une conscience constante du cadrage, sans cesse il sort du cadre et y rentre [...]. Et c'était la deuxième tentative que nous faisions : à part cette relation, la conscience qu'a l'acteur de la caméra (il pouvait regarder la caméra s'il le voulait), il fallait aussi provoquer une stimulation mutuelle entre l'acteur et la caméra, entre les acteurs, et entre cet ensemble et moi, et nous travaillions la situation en contrepoint de ce qui était montré, c'est-à-dire que nous escamotions un peu le centre général de l'attention en le laissant un peu hors du champ, ce qui créait deux centres d'intérêt, celui que nous voyions, et celui qui restait 〈off〉.» $\mathbf{2 6}$

Le principe de l'ellipse généralisée, qui régule l'avancée chaotique et la soudaine suspension du récit, est conditionné par une contrainte formelle, qui transpose, sur un plan filmique, le trope de la métonymie : l'un des centres d'intérêt du plan, avec lequel interagit l'acteur, est rejeté hors-champ; l'acteur, abstrait du milieu dans lequel il s'inscrit, est sommé de répondre à la présence insinuante de la caméra, et de réagir parallèlement aux dérobades imprévisibles de l'opérateur décadrant et recadrant sans cesse la scène. En somme, la bonne intelligibilité du film est déjà contrecarrée par la lecture malaisée des plans, ceux-ci étant polycentrés et instables: le premier long-métrage de Ruiz s'apparente ainsi à un film de "laboratoire» qui éprouve différentes figures de style et modes narratifs; un certain nombre d'aspects, en l'occurrence la réduction des personnages à des masques sociaux, l'ambiguïté du mode de référentialité des séquences et l'irrésolution du récit, sera systématisé
24 Un objectif grand angle ou à courte focale comme le $28 \mathrm{~mm}$, couramment utilisé à la fin des années 1960, favorise les plans moyens, tout en accentuant les perspectives. Nous ne pouvons par contre pas nous prononcer sur le nivellement ou au contraire l'accentuation des niveaux de contraste de la pellicule noir-blanc, à partir de la copie que nous avons visionnée (copie $35 \mathrm{~mm}$ d'époque, déposée à la Cinémathèque suisse).

25 Ruiz, à propos du travail sur le hors-champ et le décadrage: "La particularité de ce film est qu'il travaille sans cesse le décadrage, c'està-dire qu'il y a un déséquilibre constant dans le cadrage qui oblige à tenir compte du horschamp: pour saisir un cadrage, on a toujours besoin du hors-champ. " (Federico de Cardenas, "Entretien avec Raùl Ruiz (Trois tristes tigres)", op. cit., pp. 19-20).

26 ld., p. 20. 
dans les films de la maturité de Ruiz, tandis que d'autres formes d'expérimentation ne seront pas reprises, ni développées, par la suite.

\section{Cartographie politique du Chili}

Plusieurs séquences au statut d'exception, à l'instar de la scène d'entrée dans la fiction Tres Tristes Tigres, se démarquent du rythme syncopé

27 Ruiz, interrogé sur la structure narrative des pièces de théâtre qu'il a écrites au début des années 1960, oppose à la "théorie du conflit central" les "structures d'archipel de la réalité", composées de "grands espaces de silence" et de "zones de soudaine concentration d'énergie" (Benoît Peeters, "Les années chiliennes. Entretien avec Raoul Ruiz", op. cit., p. 11).

28 Ruiz, avec Tres Tristes Tigres, entend "montrer le Chili": "Et le montrer un peu différemment des représentations stéréotypées qu'on en donnait souvent. Je voulais notamment réagir contre un film appelé Ayudeme Usted Compadre, qui avait été soutenu par le gouvernement de Eduardo Frei et avait eu beaucoup de succès. Dans ce film, on montrait un Chili puissant et attirant, dont les habitants avaient quelques vices sympathiques comme un léger alcoolisme. Bref, on y montrait le Chili comme un pays de blancs, fréquentable. Moi, dans Trois Tristes Tigres, j'ai voulu présenter un tout autre Chili. Par exemple j'ai tenu à montrer les gens avec leur accent. II y avait donc une volonté pour ainsi dire politique de montrer les accents, les comportements quotidiens, les manières d'être de la rue." (Id., pp. 18-19). Notons que Tres Tristes Tigres n'a fait que 50000 entrées au Chili, tout en remportant le Prix de la critique dans ce pays.

29 "Je savais que mon film serait un des premiers au Chili à prendre ses protagonistes dans une classe qui est une majorité et je voulais que le spectateur s'y reconnaisse [...]. C'est une série de petits détails qui vont les caractériser et qui font partie de la vie de toute cette grande partie de notre population vivant au jour le jour, occupée à de petits emplois qui lui permettent juste de survivre. De là vient que la frontière légalité-illégalité, permis-interdit est très étroite et se perd. C'est qu'elle n'a aucune importance face à ce qui est en jeu. Ceci est une explication limitée de la double vie des personnages du film, mais c'est la seule qui puisse être avancée." (Federico de Cardenas, "Entretien avec Raùl Ruiz (Trois tristes tigres)", op. cit., p. 18). du film, rompant avec la logique du coq à l'âne auquel semble parfois répondre le récit - ou, plus exactement, rompant avec une écriture surdéterminée dont les clefs d'interprétation ont été effacées, scellées sous la trame apparente du film. Tout se passe comme si, malgré le brouillement des pistes, quelques blocs de plans condensaient le propos, formant des «îlots d'archipel» $\mathbf{2 7}$ qui résistent à la stratégie d'éclatement du texte filmique. Trois échanges de paroles infléchissent le film en direction d'une lecture politique : celui-ci s'apparente, localement et ponctuellement, à une entreprise de cartographie politique du Chili. Car, en un sens, c'est bien de cela dont il s'agit: Tres Tristes Tigres, conçu en réaction à Ayúdeme Usted Compadre (Aidez-moi compère, Germán Becker, Chili, 1967), film adoubé par le gouvernement d'Eduardo Frei 28, entend mettre l'accent sur la situation économique et sociopolitique du Chili, en se concentrant sur des personnages ordinaires, de la classe sociale populaire 29 - cet ancrage dans la réalité sociale, politique et économique du pays relativisant par ailleurs la dimension allégorique et la propension à symboliser de Tres Tristes Tigres.

Un dialogue énigmatique s'engage entre Tito et Luis, suite à la scène du meurtre, singulièrement dédramatisée - dans une boîte de nuit, à la fin d'un numéro de strip-tease, une altercation éclate entre un client et Tito; Tito demande lapidairement: "passe-moi le surin"; le client, après s'être exclamé: «il n'a pas pu le faire seul», s'effondre, alors que retentissent les acclamations du public saluant la fin du spectacle; du sable est répandu sur le sol pour masquer les traces de sang. Tito et Luis s'engagent dans un couloir, la caméra les suivant, à travers deux plans séquence. Tito, recadré en plan rapproché, adossé à un mur, rapporte l'histoire de Don Gomez, conseiller municipal: celui-ci, malade, se rend à Paris pour consulter les meilleurs médecins; le "grand patron" lui confie que le seul remède est de se rendre au fin fond de l'Amérique du Sud, dans un pays qui s'appelle le Chili; sur une carte, en traçant une croix à travers le pays, on trouve à l'intersection La Puntilla ou San Jose de la Punta. La caméra, à travers un autre axe de prise de vues, suit Tito qui poursuit son récit: Don Gomez aurait répondu qu'il vient de ce lieu-dit. Tito donne alors un objet à Luis, avant de quitter les lieux: Luis découvre un couteau enveloppé dans le journal que lui a remis 
Tito. Le montage de la séquence est tout à fait remarquable sur le plan de la construction du récit: il est malaisé de retracer les évènements qui prennent place dans la boîte de nuit, l'action étant fragmentée par le cadrage rapproché et les coupes sèches; ce n'est qu'à travers le dernier plan que nous avons décrit, réinscrivant le surin, que le spectateur peut reconstituer l'action qui s'est déroulée. Les propos de Tito et Luis, mobilisant la structure du récit allégorique, évident la fable rapportée de son sens second, métaphorique : faut-il comprendre par là qu'il ne sert à rien de fuir son pays, que nos origines toujours nous rattrapent? $\mathrm{Ou}$ au contraire que l'appartenance à la culture latino-américaine, chilienne, constitue une malédiction à laquelle on ne peut échapper? Le motif de la carte géographique, et plus précisément le tracement d'une croix sur celle-ci, désignant la croisée des chemins, doivent-ils être reliés à la scène du meurtre? Autant de questions laissées en suspens...

La deuxième séquence "allégorique» constitue une variation sur la notion de cartographie, mais cette fois à travers une scénarisation qui évoque lointainement les tableaux vivants. Dans un café jonché de bouteilles vides, Luis décrit à Amanda le paysage que celles-ci reflètent, lorsqu'elles sont éclairées par un projecteur improvisé que manie un technicien. Disposant deux bouteilles en croix, Luis regarde à travers celles-ci, en évoquant face à Amanda différents monuments ou attractions touristiques du Chili que le miroitement intermittent du verre laisse entrevoir (la gare Mapocho, la commune d'Independencia, le palais de La Moneda et la colline San Cristobal à Santiago, ou encore la Cordillière des Andes). Luis, en dirigeant le faisceau du projecteur sur la scène, fait surgir ces villes imaginaires en disposant les bouteilles selon un ordre connu de lui seul ("Deux au Far West et une autre au coin»). Le territoire imaginaire ou plutôt l'imaginaire du territoire que le personnage laisse apparaître, dans cette séquence qui met en abyme le film et son dispositif de tournage, est indissociable du Chili et de son histoire; mais plutôt que de proposer une vision officielle du pays, Ruiz l'inscrit en creux, en faisant retour sur une réalité plus concrète et triviale, en l'occurrence la vie nocturne des cafés.

La dernière de ces séquences articule indéniablement un propos politique. Dans la salle d'attente des autocars qui sillonnent le pays, un inspecteur interroge Tito sur son ami Luis qui s'apprête à quitter la ville. Les premières questions du représentant de l'ordre portent sur les affaires qui se sont tramées autour de Rudi; mais rapidement, il accuse Tito d'avoir abandonné son compagnon. Enjoignant Tito à boire à sa santé, l'inspecteur lui rapporte une fable, qui pourrait s'avérer menaçante. Les voyages à Santiago sont désormais réguliers; son ami Luis, que Tito a 
30 Ruiz cite comme modèle le poème "Soliloque de l'individu" de Nicanor Parra ("Soliloquio del individuo", Poemas y antipoemas, Nascimento, Santiago, 1954 [traduction partielle dans Esprit, $\mathrm{n}^{\circ} 7$, juillet 1986]). Voir Benoît Peeters, "Les années chiliennes. Entretien avec Raoul Ruiz", op. cit., p. 18.

31 Ruiz s'attache à définir une forme de polysémie visuelle qui serait spécifique au cinéma et aux images hypnagogiques, à travers la notion d'“image-situation" ou de "double image": "Reste que cette double image n'est possible que sous certaines conditions: 1) ne pas être interprétée comme deux images superposées, dont l'une serait réelle et l'autre symbolique: la femme fatale superposée à un serpent (à moins que la femme fatale ne collectionne les serpents et qu'à la fin du film elle périsse, étranglée par un reptile); 2) les deux images superposées devraient faire référence à une troisième, invisible mais évidente: la femme fatale et le serpent font référence à l'errance des âmes: d'une bête à un homme et vice versa; 3) l'image doit éviter de se constituer en allégorie autonome. Elle doit demeurer fragmentaire, inachevée." (Raoul Ruiz, Poétique du cinéma 1, op. cit., p. 112). abandonné à son sort sans état d'âme, est devenu une brebis galeuse, car il est tombé entre les mains de personnes qui lui demandaient beaucoup d'argent; il a abandonné sa famille ainsi que la coopérative, ce qui est bien plus grave; d'autant que pour l'instant, c'est l'inspecteur qui gère la coopérative "Voyage à Santiaguito". Cette séquence, renvoyant aux interrogatoires politiques tout en se déroulant dans un lieu d'attente, à la vue et au sus de tout un chacun, évoque le sort réservé aux dissidents dans le pays, tout en introduisant à nouveau un glissement de signifiants (Santiago/Santiaguito - c'est-à-dire une ville au Mexique célèbre pour son architecture coloniale ou encore un volcan au Guatemala dont les éruptions sont meurtrières).

\section{Errances}

Dans une perspective diamétralement opposée, il est possible de soutenir que Tres Tristes Tigres met en scène de simples errances: dès l'ouverture du film, qui se présente sous forme d'un premier va-et-vient sans véritable destination, le récit récuse toute progression linéaire. Un premier plan séquence s'articule autour d'Amanda qui hèle Tito, assis dans un autocar sur le point de quitter la ville; elle demande ainsi à un passager un crayon pour noter son adresse, alors que le chauffeur s'impatiente. Lors du plan qui suit, raccordant mal avec le précédent, le spectateur découvre Tito, dans la rue, qui présente son ami Luis à Amanda; les trois personnages prennent un taxi, alors que l'autocar est déjà au loin. Enfilade de situations improbables, rencontres et heurts entre les corps, dissolution de l'espace intime sous la pression de la sphère publique, gestes dictés par l'absorption régulière d'alcool, le film est construit à l'image du fourchelangue du titre: les calembours visuels, associés à une perte de contrôle des situations, déterminent l'enchaînement des péripéties. En un sens, c'est bien ce qu'indique Ruiz lorsqu'il soutient que le poète Nicanor Parra, qui manie avec virtuosité les jeux de mots, les ambiguïtés de langage et les effets d'autoréférence, constitue l'une des principales sources d'inspiration de Tres Tristes Tigres ${ }^{30}$.

On le sait, les films de Ruiz se cristalliseront par la suite autour d'«images-situation» $\mathbf{3 1}$, de séquences-palimpseste où plusieurs récits virtuels ou actualisés se superposent, les formes donnant lieu à une double, voire à une triple lecture. A la différence de la logique de l'anamorphose et des structures en trompe-l'œil, tout se passe comme si, dans Tres Tristes Tigres, une succession d'instants présents et d'espaces quelconques contrecarrait le déroulement du récit et grevait d'incomplétude les plans. Tres Tristes Tigres, on l'a dit, est découpé en deux parties distinctes: la seconde partie est introduite par des plans sur une rue, puis par une séquence 
filmée depuis un taxi; les personnages s'éveillent, autour de midi (Tito, dans une chambre d'hôtel, en découvrant le mot laissé par sa compagne d'un soir; Luis et Amanda, s'extirpant des brumes de l'alcool, dans une autre chambre). De plus, la structure d'ensemble du film est quelque peu circulaire, à l'image du renversement du rôle des personnages d'une partie à l'autre (victime/bourreau). Néanmoins, les différentes pièces du puzzle peinent à s'imbriquer: chaque séquence est dotée d'une certaine autonomie; elle raccorde mal avec un dessein général. D’ambiance résolument nocturne, le film saisit des corps désœuvrés; le bar clandestin puis la boîte de nuit où se nouent les accords immobiliers entre Rudi et l'homme d'affaires Carlos, frappent d'irréalité ces scènes censément orientées par l'action; de la même façon, les premières discussions entre Tito, Amanda et Luis autour de ces activités illicites empruntaient le biais de la métaphore culinaire, comme à travers un langage codé. L'incertitude est le seul principe auquel le spectateur peut se rattacher: à quel personnage avons-nous affaire? Quel geste, quelle tâche, est-il en train d'effectuer? Le centre autour duquel le film semble graviter est laissé vacant: Tres Tristes Tigres accumule les récits enchâssés et les digressions impromptues, sans jamais les reprendre en une structure d'ensemble, en une logique forclose.

Au terme de ce parcours, quels éléments pouvons-nous inférer au sujet de l'œuvre encore en gestation de Ruiz? En premier lieu, la structure de l'anamorphose et du trompe-l'œil ne conditionne pas l'univers filmique de Ruiz, qui échappe à toute stratégie systématique et unifiée : le cinéaste fait feu de tout bois, ajuste à vue ses dispositifs et détourne les contraintes qu'il s'impose (ou qui lui sont imposées) par esprit ludique ; la logique de Ruiz s'apparente à la structure ouverte et imprévisible du jeu (play), à un espace potentiel d'échanges que Winnicott distingue radicalement des jeux à règles définies $($ game $)$ 32. En second lieu, l'«élan allégorique» qui conditionne l'œuvre baroque constitue chez Ruiz un point de fuite auquel tend un certain nombre de films sans pour autant s'y conformer: pour que l'étourdissement, la perte de repères, ait lieu, il faut que le récit suive obstinément un point aveugle sans cesse approché, jamais atteint, entraînant d'improbables détours, suivant la rhétorique du paradoxe (d'où la centralité de la référence à Klossowski et au Baphomet dans ce jeu d'énigmes insolubles $\left.{ }^{33}\right)$. Tres Tristes Tigres s'inscrit en amont de ces deux horizons de référence ou d'attente. Le film ne se réduit pas à un tortueux labyrinthe dont l'architecture suivrait les plans d'un mystérieux codex; il ne se constitue pas en une parabole scolastique qui délivrerait un savoir oublié sur les affaires politiques de ce monde. Ce premier long métrage s'inscrit dans le «style international
32 Voir D. W. Winnicott, Jeu et réalité. L'espace potentiel, Gallimard, Paris, 1975 [Playing and Reality, Basic Books, New York, 1971].

33 Ruiz adapte en 1977 le premier roman de Pierre Klossowski (La Vocation suspendue, Gallimard, Paris, 1950); I'année suivante, il réalise L'Hypothèse du tableau volé, film vertigineux qui prend pour point de départ Le Baphomet (Mercure de France, Paris, 1965). Ce roman cryptique de Klossowski constitue peut-être l'une des clefs d'accès privilégié pour qui veut pénétrer l'univers retors et miroitant de la fiction ruizienne. 
34 En architecture, le style international renvoie aux mouvements modernistes qui ont essaimé entre les années 1920 et les années 1960, dans la filiation du Bauhaus et de l'Esprit nouveau, en privilégiant le fonctionnalisme et les matériaux nouveaux (voir Henry-Russel Hitchcok, Philip Johnson, The International Style, W. W. Norton, New York/Londres, 1966 [première édition: "The International Style: Architectur since 1922", in Modern Architectur, 1932]; Hasan-Uddin Khan, Le Style international. Le modernisme dans l'architecture de 1925 à 1965, Taschen, Köln, 1995). Par analogie, les nouveaux cinémas qui se développent à partir de la fin des années 1950 peuvent être considérés comme un mouvement international qui repose sur une épuration du style et un mode de récit ouvert, exploitant tantôt les plans séquence longuement tenus, tantôt un montage elliptique et discontinu. moderniste $\mathbf{3 4}$ des années 1960, tout en disloquant un certain nombre de traits caractéristiques qui formeront bientôt système. Le choix délibéré du plan moyen contredit la maîtrise associée au plan séquence (et le prétendu respect de "l'ambiguïté du réel» à travers le creusement de la profondeur de champ); les mouvements instables de la caméra tenue à l'épaule court-circuitent toute stratégie d'exhibition des marques d'énonciation (et l'«effet de distanciation" qu'induiraient le montage discontinu et les faux raccords). Le travail sur la forme, le style, et sur l'aléa, l'accident, est contrecarré par le recours à une logique "nonsensique» qui laisse les tensions avivées et le récit indéterminé. 